\title{
Untangling Electrostatic and Strain Effects on the Polarization of Ferroelectric
}

\section{Superlattices}

Ekaterina Khestanova, Nico Dix, Ignasi Fina, Mateusz Scigaj, José Manuel Rebled, César

Magén, Sonia Estradé, Francesca Peiró, Gervasi Herranz, Josep Fontcuberta, and Florencio

Sánchez ${ }^{*}$

E. Khestanova, N. Dix, Dr. I. Fina, M. Scigaj, J.M. Rebled, Dr. G. Herranz, Prof. J.

Fontcuberta, Dr. F. Sánchez

Institut de Ciència de Materials de Barcelona (ICMAB-CSIC), Campus UAB, Bellaterra

08193, Barcelona, Spain

Email: fsanchez@icmab.es

M. Scigaj

Dep. de Física, Universitat Autònoma de Barcelona, Campus UAB, 08193 Bellaterra, Spain

J.M. Rebled, Dr. S. Estradé, Dr. F. Peiró

Laboratory of Electron Nanoscopy (LENS-UB), Institute of Nanoscience and

Nanotechnology (In2UB), Electronics Department, University of Barcelona, c/ Martí i

Franqués 1, Barcelona 08028, Catalonia, Spain

Dr. C. Magén

Laboratorio de Microscopías Avanzadas (LMA), Instituto de Nanociencia de Aragón (INA) ARAID, and Departamento de Física de la Materia Condensada, Universidad de Zaragoza, Zaragoza 50018, Spain

Keywords: thin films; epitaxy; oxides; ferroelectric superlattices; $\mathrm{BaTiO}_{3}$ 
The polarization of ferroelectric superlattices is determined by both electrical boundary conditions at the ferroelectric/paraelectric interfaces and lattice strain. The combined influence of both factors offers new opportunities to tune ferroelectricity. However, the experimental investigation of their individual impact has been elusive because of their complex interplay. Here, we present a simple growth strategy that has permitted to disentangle both contributions by an independent control of strain in symmetric superlattices. It is found that fully strained short period superlattices display a large polarization whereas a pronounced reduction is observed for longer multilayer periods. This observation indicates that the electrostatic boundary mainly govern the ferroelectric properties of the multilayers whereas the effects of strain are relatively minor.

\section{Introduction}

Superlattices combining ferroelectric and paraelectric nanometric layers are artificial materials in which electrostatic coupling can induce polarization in the paraelectric material. ${ }^{[1-}$ ${ }^{3]}$ A plethora of exciting properties have been observed in ferroelectric superlattices, including ferroelectricity in layers only one unit cell thick, ${ }^{[4,5]}$ polarization enhancement, ${ }^{[5-8]}$ improper ferroelectricity in $\mathrm{PbTiO}_{3} / \mathrm{SrTiO}_{3}$ superlattices, ${ }^{[9]}$ phonon interference effects, ${ }^{[10]}$ negative capacitance, ${ }^{[11]}$ ferroelectricity in superlattices that do not include a ferroelectric layer, ${ }^{[12]}$ or the stabilization of polar vortices confined in ferroelectric layers of long-period superlattices. ${ }^{[13]}$ Electrical boundary conditions in ferroelectric superlattices are critical since the ultrathin ferroelectric layers are in contact with paraelectric layers that are not effective to screen bound charges. ${ }^{[14,15]}$ Depending on the layers thickness the stray electric fields generated by the ferroelectric dipoles can be confined in/near the ferroelectric layers forming domains or more complex patterns, or they can induce polarization in the paraelectric layer 
permitting uniform polarization across the superlattice. ${ }^{[1,6,16,17]}$ On the other hand, the ultrathin thickness of the ferroelectric and paraelectric layers limits the plastic relaxation of the epitaxial strain in the superlattice and consequently, short period superlattices under compressive epitaxial stress can be fully coherent with expanded out-of-plane cell parameter and increased polarization. Thus, the superlattice period critically determines the ferroelectricity by the dual influence of mechanical (strain) and electrostatic (interfaces) boundary effects.

The electrostatic coupling in superlattices, and the influence of the superlattice period, was soon confirmed experimentally. ${ }^{[2-5,8,18]}$ Ab-initio calculations predicted that the polarization of symmetric n-BTO/n-( $\mathrm{SrTiO}_{3}$ or $\left.\mathrm{CaTiO}_{3}\right)$ superlattices ${ }^{[19-21]}$ increases with the number (n) of unit cells in the layers. ${ }^{[22]}$ However, the opposite result (lower polarization when increasing the superlattice period) was found experimentally in symmetric BTO/CaTiO 3 (CTO) superlattices. ${ }^{[23]}$ Nevertheless, as the BTO/CTO superlattices were found to be more relaxed for higher $\mathrm{n}$, both the electrostatic conditions and the strain conditions varied in the whole set of samples, thus challenging the comparison with theoretical results. Indeed, although the relative contribution of these parameters could not be isolated, it was concluded that the measured reduction of polarization when the period $n$ is increased was dominated by strain relaxation, ${ }^{[23]}$ as supported by earlier theoretical predictions. ${ }^{[19]}$ This issue, of crucial interest on the way towards engineered ferroelectric multilayers, remains unsolved. Some few open questions are: 1) to confirm experimentally the ab-initio calculations of the dependence of polarization on superlattice period, and 2) to isolate experimentally the relative influence of lattice strain and electrostatic boundary conditions on ferroelectric properties of superlattices. Aiming to contribute to solve these pending questions, here: i) we have grown fully coherent symmetric $\mathrm{Mx}(\mathrm{n}-\mathrm{BTO} / \mathrm{n}-\mathrm{STO})$ superlattices (M is the number of the BTO-STO bilayers stacked in the superlattice, and $\mathrm{n}$ is the number of unit cells in each BTO or STO layer of the stacked BTO-STO bilayers) for a wide range of period n values, and ii) we have achieved 
controlling the lattice strain in fully coherent superlattices of a fixed period by changing the deposition rate. Ferroelectric polarization loops were measured for all the superlattices, and it was found that their polarization displays a strong dependence on the period $n$ and, in comparison, a tiny dependence on the lattice strain. Thus, electrostatic boundary conditions in multilayers rule the ferroelectric response, whereas the lattice strain plays a relatively minor role.

\section{Results and Discussion}

Fabrication of superlattices requires two-dimensional growth of the layers and accurate control of the thickness of the individual layers. A 10x(4 u.c.-BTO/4 u.c. -STO) superlattice, sketched in Figure 1a, was prepared on a $\mathrm{STO}(001)$ substrate buffered with a $10 \mathrm{~nm}$ thick $\mathrm{La}_{0.67} \mathrm{Sr}_{0.33} \mathrm{MnO}_{3}$ (LSMO) electrode. The reflection high energy electron diffraction (RHEED) pattern (Figure 1b) recorded along LSMO[100] displays bright specular and Bragg spots positioned in the $0^{\text {th }}$ Laue zone, signaling that the LSMO film is epitaxial and flat. BTO and STO were then alternatively deposited to obtain the 10x(4-BTO/4-STO) superlattice, being BTO the first layer grown on the LSMO electrode. The dependence of the intensity of the specular RHEED spot with time is shown in Figure 1c. The intensity data during BTO and STO deposition are plotted in blue and red, respectively, whereas the data when switching between BTO and STO deposition are plotted in black. The ten sequences corresponding to the $10 x(4-B T O / 4-S T O)$ superlattice are appreciated, with an overall reduction of RHEED intensity during BTO deposition and an overall increase when STO grows. The intensity oscillations are persistent, as can be seen for the $10^{\text {th }}$ stack in Figure $1 \mathrm{~d}$. The first four intensity oscillations, plotted in blue, correspond to the four monolayers of BTO and present a moderate amplitude damping. The zoom in Figure 1e shows quick intensity reduction with the arrival of atoms after each laser pulse followed by intensity recovery. Further intensity recovery when the laser was stopped at the maxima of the fourth oscillation signals surface 
smoothing. Finally, the last stack of four STO monolayers was deposited and high amplitude oscillations were observed (see the red curve in Figure 1d and the zoom in Figure 1f), accompanied by a progressive increase of the average intensity. The RHEED pattern in Figure 1g, recorded along STO[100] at high temperature just at the end of the deposition, attests epitaxy and the spots in the $0^{\text {th }}$ Laue zone suggest a flat surface.

The quality of the $10 x(4-\mathrm{BTO} / 4-\mathrm{STO})$ superlattice was investigated by aberration corrected scanning transmission electron microscopy (STEM). Figure 2a shows composition maps ( $\mathrm{Ti}, \mathrm{Ba}, \mathrm{Mn}$, and $\mathrm{La}$ ) of a narrow region along the growth direction determined by electron energy loss spectroscopy (EELS) in STEM. High-angle annular dark field (HAADF) and color composition images are in the left and the right of Figure 2a, respectively. The compositional abruptness in the interfaces discards chemical interdiffusion. A HAADFSTEM image of a wider region is presented in Figure $2 \mathrm{~b}$, and a region including the STO substrate, the LSMO electrode, and the first superlattice stacked layers is zoomed in Figure 2e. The LSMO film is around $10 \mathrm{~nm}$ thick, and the superlattice has a total thickness of around 32 $\mathrm{nm}$ as expected by the RHEED oscillations. STO layers are darker in the images, whereas LSMO and BTO layers are brighter due to the high atomic number of La and Ba, respectively. Absence of dislocations and analysis of selected area diffraction images (not shown) signal fully coherent growth. The HAADF-STEM images in Figures $2 b$ and $2 e$ were used for Geometrical Phase Analysis (GPA) to obtain strain maps, using the STO substrate as reference. The corresponding in-plane strain maps are plotted in Figures 2c and 2f, and the out-of-plane strain maps are plotted in Figures 2d and 2g. There is no measurable in-plane strain in the LSMO film and the BTO and STO layers respect to the STO substrate, confirming fully coherent growth of the superlattice. In contrast, the maps along the out-ofplane direction show clear contrast, being the deformation with respect to STO around $-2.1 \%$ in the LSMO layer and around $6.8 \%$ in the BTO layers. 
Aiming to compare the effects of the number of interfaces and lattice strain, we fabricated two series of Mx(n-BTO/n-STO) superlattices, with fixed total thickness $(\mathrm{Mx} 2 \mathrm{n}=$ 120 u.c., corresponding to around $48 \mathrm{~nm})$, on $\mathrm{TiO}_{2}$-terminated $\mathrm{STO}(001)$ substrates. The growth rate and superlattice geometry of the samples are summarized in Table I. In Series I, six superlattices were grown at the rate of $0.30 \AA$ A pulse, and the number of interfaces varied from $11(M=6, n=10)$ to $119(M=60, n=1)$. In Series II, with the number of interfaces fixed to $19(M=10, n=6)$, four superlattices were deposited at growth rate of $0.07,0.17,0.30$ and 0.53 Å/pulse. The $10 x(6-B T O / 6-S T O)$ superlattice deposited at $0.30 \AA$ A pulse is common to both series.

Atomic force microscopy (AFM) topographic images of the samples of series I and II are presented in Figure 3. Label at the top of each image indicates the superlattice geometry ( $\mathrm{M}$ and $\mathrm{n}$ ) and the growth rate. Remarkably, all superlattices are extremely flat and present morphology of terraces and steps 1 u.c. high, even the one grown at the highest rate $(0.53$ Å/pulse), the one with thickest BTO and STO layers (10 u.c. thick each), and the one with higher amount of interfaces (119 BTO/STO interfaces in the 60x(1-BTO/1-STO) superlattice). It is also noticeable that morphology of terraces and steps occurs on substrates having very different miscut angles, ${ }^{[24]}$ as indicated by the spread of terrace widths ranging from around 80 to around $400 \mathrm{~nm}$. In some of the samples the steps are straight over large distances, whereas in other superlattices, particularly in those on high miscut angle substrates, steps present a higher density of kinks. Also, some dislocation etching pits (square holes few tens of nm wide), caused by the chemical etching to obtain single $\mathrm{TiO}_{2}$-termination, are observed in most of the images.

Figure 4a shows the specular X-ray diffractometry (XRD) $\theta-2 \theta$ scans of the four 10x(6-BTO/6-STO) superlattices prepared at different growth rate (Series II). In addition to the substrate and LSMO peaks, there are superlattice reflections. The zoom at low angles (Figure 4b) shows Laue peaks around the superlattice reflections, whereas in the high angles 
zoom (Figure 4c) a shift in the angular position of the reflections between the samples can be appreciated, being the peaks shifted to lower angles as higher is the growth rate. It indicates that the out-of-plane parameter increases with the growth rate. The XRR curves of these samples are in Figure 4d, showing Kiessig fringes and superlattice reflections. The XRD reciprocal space map (RSM) in Figure 4g corresponds to the superlattice grown at a rate of $0.30 \AA$ Apulse (the sample that belongs to both Series I and Series II). The RSM, around the asymmetrical STO(103) reflection, shows several superlattice reflections matching the same $\mathrm{Q}_{\mathrm{x}}$ coordinate of the substrate reflection, indicating that the superlattice is fully strained as locally observed in the superlattice characterized by STEM. The samples deposited at lower growth rate are also fully strained (the RSM corresponding to the sample grown at 0.07 $\AA$ /pulse is in Figure 4h). The $\theta-2 \theta$ scans of the superlattices with different number of interfaces (Series I) deposited at the same rate of $0.30 \AA$ Apulse are presented in Figure 4e. The zoom at high angles (Figure 4f) shows that (008n) superlattice reflections ( $\mathrm{n}$ is the superlattice period) are at different angles, and thus indicates that the out-of-plane lattice parameter depends on the superlattice period.

The out-of-plane lattice parameter of BTO was estimated from the specular XRD $\theta-2 \theta$ scans, assuming that the STO layers have bulk $c$-axis (see in Supporting Information the calculated parameter considering expansion of the STO unit cell). In Figure 5a the BTO caxis is plotted as a function of the BTO layers thickness (Series I). The $c$-axis length increases monotonically from $4.149 \AA$ for the thinnest BTO (n=1) to $4.234 \AA$ for the superlattice with n = 6. The corresponding unit cell expansion $(\varepsilon)$ respect bulk BTO (4.038 $\AA$ ) is very high, up to $\varepsilon=4.9 \%$. However, in the superlattice with the thickest layers $(n=10)$ the expansion of the $c$ parameter is reduced to $4.201 \AA$, probably due to partial plastic relaxation. The dependence of the $c$-parameter with the growth rate is plotted in Figure 5b, showing a monotonic increase from $4.188 \AA$ ( $\varepsilon=3.7 \%)$ to $4.258 \AA$ ( $\varepsilon=5.4 \%)$. The influence of the deposition rate points to 
the relevance of growth kinetics, suggesting that there are less defects contributing to the $c$ axis expansion if the superlattice grows slowly. The influence of growth kinetics in the formation of defects and its control by either laser fluence or growth rate has been also discussed recently for $\mathrm{BTO}^{[25]}$ and $\mathrm{STO}^{[26]}$ single films, respectively.

The room-temperature polarization loops of the superlattices of Series I (n from 1 to 10) are shown in Figure 6a. The remnant polarization $\mathrm{P}_{\mathrm{r}}$ is plotted against the superlattice period $\mathrm{n}$ in Figure 5a (solid circles). All the samples display switchable ferroelectric polarization, and even the $\mathrm{n}=1$ superlattice shows a remnant polarization $\mathrm{P}_{\mathrm{r}}$ of around 2.9 $\mu \mathrm{C} / \mathrm{cm}^{2}$ (with spontaneous polarization $\mathrm{P}_{\mathrm{s}}$ around $6 \mu \mathrm{C} / \mathrm{cm}^{2}$ ). The $\mathrm{n}=2$ sample has the highest polarization, with $\mathrm{P}_{\mathrm{r}}$ close to $22 \mu \mathrm{C} / \mathrm{cm}^{2}$ and $\mathrm{P}_{\mathrm{s}}$ above $30 \mu \mathrm{C} / \mathrm{cm}^{2}$. The other samples of the series show loops with lower $\mathrm{P}_{\mathrm{r}}$ as the period of the superlattice increases, presenting the $\mathrm{n}=$ 10 sample $\mathrm{P}_{\mathrm{r}}$ around $2.9 \mu \mathrm{C} / \mathrm{cm}^{2}$. The graph evidences the reduction of the ferroelectric polarization with the superlattice period $n$, beyond the singular $n=1$ superlattice. The polarization loops of the $10 x(6-\mathrm{BTO} / 6-\mathrm{STO})$ superlattices deposited with different growth rate are shown in Figure 6b. Differences between loops are clearly smaller than those in Figure 6a, indicating that the growth rate has lower influence on the ferroelectric polarization loops that the number of interfaces. The dependence of $\mathrm{P}_{\mathrm{r}}$ with growth rate (Figure $5 \mathrm{~b}$, empty circles) shows moderately lower $\mathrm{P}_{\mathrm{r}}$ (from around 8.6 to $6 \mu \mathrm{C} / \mathrm{cm}^{2}$ ) as higher is the growth rate (from 0.07 to $0.53 \AA$ A/pulse), whereas the $c$-axis of BTO (Figure 5b, empty triangles) showed a monotonic increase from $4.188 \AA(\varepsilon=3.7 \%)$ to $4.258 \AA(\varepsilon=5.4 \%)$. The data of this Series (superlattices deposited at different growth rate) are also plotted in Figure 5a (empty circles and triangles for $\mathrm{P}_{\mathrm{r}}$ and $c$-axis parameter data, respectively) together with the values corresponding to Series I (superlattices with different number of interfaces). It is appreciated that high polarization is not due to large $c$-axis parameter, as clearly observed in Figure $5 c$ where the remnant polarization of the superlattices of both series is plotted against the $c$-axis 
of BTO. Therefore, it is demonstrated the ruling influence of electrical boundary conditions in comparison with BTO lattice parameter on the ferroelectric polarization.

The observed correlation of the ferroelectric polarization with the superlattice period is a consequence of the dominating influence of electrostatic boundary conditions. The spontaneous polarization measured in the superlattices is plotted against the period $\mathrm{n}$ in Figure $5 d$ (solid circles). The $\mathrm{n}=2$ superlattice, with high $\mathrm{P}_{\mathrm{r}}=22 \mu \mathrm{C} / \mathrm{cm}^{2}$ and $\mathrm{P}_{\mathrm{s}}=30.4 \mu \mathrm{C} / \mathrm{cm}^{2}$ likely presents uniform polarization across the BTO and STO layers. Increasing the period to $n=10$ the reduction in polarization $\left(\mathrm{P}_{\mathrm{r}} \approx 2.9 \mu \mathrm{C} / \mathrm{cm} 2\right.$ and $\mathrm{P}_{\mathrm{s}} \approx 7 \mu \mathrm{C} / \mathrm{cm}^{2}$ ) would be the result of domain formation in the individual BTO layers due to the high energy cost of polarizing thick paraelectric layers. ${ }^{[16-18]}$ Figure 7 sketches the polarization distribution as the period increases from $n=2$ to more than $n=10$. The conclusion from our experimental observation agrees with theoretically calculations ${ }^{[16]}$ that considered epitaxially strained 2-BTO/2-STO and 10BTO/10-STO superlattices. Indeed, Lisenkov and Bellaiche ${ }^{[16]}$ concluded that under compressive epitaxial strain similar to the existing in the superlattices we have investigated, the $n=2$ superlattice presents at room temperature a uniform ferroelectric phase, whereas the $\mathrm{n}=10$ superlattices develops an unswitchable closed domain structure. The transition between uniform polarization and a polydomain phase was recently predicted ${ }^{[17]}$ and experimentally observed ${ }^{[18]}$ in $\mathrm{PbTiO}_{3}$-STO superlattices. The size of domains in BTO-STO superlattices has been experimentally determined by using synchrotron $\mathrm{XRD} .{ }^{[27]}$ The measurement of the evolution of domain size with the superlattice period could give a direct evidence of the transition from the uniform ferroelectric phase to the closed domain structure in BTO-STO superlattices.

\section{Conclusion}

In conclusion, we have investigated ferroelectric superlattices having different period and lattice strain. It has been shown that for a fixed superlattice period, the lattice parameter can be modified by adjusting the growth rate. The relative influence of interfaces and strain on the 
ferroelectric properties has been discriminated, and it is found that electrostatic boundary conditions fully dominate the ferroelectric response of the superlattices. The high polarization in short period superlattices associated to uniform ferroelectricity decreases quickly as the period increases and the cost to polarize the paraelectric STO becomes excessive. Thus, a uniform ferroelectric entity, artificial-like ferroelectric material, is limited exclusively to ultrashort period superlattices.

\section{Experimental Section}

BTO/STO superlattices were fabricated on STO(001) buffered with a LSMO electrode by RHEED-assisted PLD using a KrF excimer laser. The superlattice and the electrode were deposited in a single process, being BTO the first layer in the superlattice. LSMO was grown at substrate temperature of $725^{\circ} \mathrm{C}, 0.1$ mbar oxygen pressure and laser frequency of $2 \mathrm{~Hz}$, whereas the corresponding parameters for both BTO and STO were $700{ }^{\circ} \mathrm{C}, 0.02$ mbar and 1 Hz. The abruptness of the interfaces and the lattice strain of a 10x(4-BTO/4-STO) was investigated by HAADF-STEM and STEM-EELS in a probe-corrected FEI Titan 60-300 operated at $300 \mathrm{kV}$ and equipped with a high-brightness Schottky Field Emission Gun (XFEG), a CEOS aberration corrector and a Tridiem 866 ERS image filter/spectrometer from Gatan. A series (Series I) of six Mx(n-BTO/n-STO) superlattices having same total thickness $(\mathrm{Mx} 2 \mathrm{n}=120$ unit cells) were fabricated. In these superlattices $\mathrm{n}$ was $1,2,3,4,6$, and 10, being the corresponding number of BTO/STO interfaces 119, 59, 39, 29, 19, and 11 . The growth rate of both BTO and STO was $0.30 \AA$ À/pulse. Other three 10x(6-BTO/6-STO) superlattices were prepared changing the aperture size of the mask used to collimate the laser beam, thus determining the energy of the laser beam focused on the target. This method permits adjusting the growth rate with fixed laser fluence. The four 10x(6-BTO/6-STO) superlattices in the series were grown at $0.07,0.17,0.30$, and $0.53 \AA$ /pulse (ablation rate was the same for BTO and STO). All [Mx(n-BTO/n-STO)]/LSMO samples of Series I and II were 
grown on $\mathrm{TiO}_{2}$-terminated $\mathrm{STO}(001)$ substrates. ${ }^{[19]}$ The surface morphology was characterized by AFM. The crystal quality and the superlattice spacing was investigated by specular XRD $\theta-2 \theta$ and XRR scans. RSM around asymmetrical reflections of some superlattices were also measured to determine the in-plane lattice parameter. Ferroelectric polarization loops were determined at room temperature in top-top configuration, using a TFAnalyser2000 platform (aixACCT Systems GmbH).

\section{Supporting Information}

Supporting Information is available from the Wiley Online Library or from the author.

\section{Acknowledgements}

Financial support by the Spanish Government [Projects MAT2014-56063-C2-1-R and MAT2013-41506] and Generalitat de Catalunya (2014-SGR-734 and 2014-SGR-672) is acknowledged. ICMAB-CSIC authors acknowledge financial support from the Spanish Ministry of Economy and Competitiveness, through the "Severo Ochoa" Programme for Centres of Excellence in R\&D (SEV- 2015-0496). IF acknowledges Juan de la Cierva Incorporación postdoctoral fellowship (IJCI-2014-19102) from the Spanish Ministry of Economy and Competitiveness. The transmission electron microscopy works have been conducted in the Laboratorio de Microscopias Avanzadas at Instituto de Nanociencia de Aragon (Universidad de Zaragoza). Authors acknowledge the LMA-INA for offering access to their instruments and expertise. We thank Massimiliano Stengel for useful discussions.

[1] J.B. Neaton, K.M. Rabe, Appl. Phys. Lett. 2003, 82, 1586.

[2] M. Dawber, C. Lichtensteiger, M. Cantoni, M. Veithen, P. Ghosez, K. Johnston, K.M. Rabe, J.M. Triscone, Phys. Rev. Lett. 2005, 95, 177601. 
[3] M. Dawber, N. Stucki, C. Lichtensteiger, S. Gariglio, P. Ghosez, J.M. Triscone, Adv. Mater. 2007, 19, 4153.

[4] D.A. Tenne, A. Bruchhausen, N.D. Lanzillotti-Kimura, A. Fainstein, R.S. Katiyar, A. Cantarero, A. Soukiassian, V. Vaithyanathan, J.H. Haeni, W. Tian, D.G. Schlom, K.J. Choi, D.M. Kim, C.B. Eom, H.P. Sun, X.Q. Pan, Y.L. Li, L.Q. Chen, Q.X. Jia, S.M. Nakhmanson, K.M. Rabe, X.X. Xi, Science 2006, 313, 1614

[5] S.S.A. Seo, J.H. Lee, H.N. Lee, M.F. Chisholm, W.S. Choi, D.J. Kim, J.Y. Jo, H. Kim, J. Yu, T.W. Noh, Adv. Mater. 2007, 19, 2460.

[6] C. Lichtensteiger, P. Zubko, M. Stengel, P. Aguado-Puente, J.M. Triscone, P. Ghosez, J. Junquera, in Oxide Ultrathin films: Science and Technology, Wiley, Weinheim, Germany, 2012.

[7] P. Zubko, S. Gariglio, M. Gabay, P. Ghosez, J.M. Triscone, Ann. Rev. Condens. Mater. Phys. 2011, 2, 141.

[8] H.N. Lee, H.M. Christen, M.F. Chisholm, C.M. Rouleau, D.H. Lowndes, Nature 2005, 433, 395.

[9] E. Bousquet, M. Dawber, N. Stucki, C. Lichtensteiger, P. Hermet, S. Gariglio, J.M. Triscone, P. Ghosez, Nature 2008, 452, 732.

[10] J. Ravichandran, A K. Yadav, R. Cheaito, P.B. Rossen, A. Soukiassian, S.J. Suresha, J.C. Duda, B.M. Foley, C.H. Lee, Y. Zhu, A.W. Lichtenberger, J.E. Moore, D.A. Muller, D.G. Schlom, P.E. Hopkins, A. Majumdar, R. Ramesh, M.A. Zurbuchen, Nat. Mater. 2014, 13, 168. [11] W. Gao, A. Khan, X. Marti, C. Nelson, C. Serrao, J. Ravichandran, R. Ramesh, S. Salahuddin, Nano Lett. 2014, 14, 5814.

[12] K. Rogdakis, J.W. Seo, Z. Viskadourakis, Y. Wang, L.F.N.A. Qune, E. Choi, J.D. Burton, E.Y. Tsymbal, J. Lee, C. Panagopoulos, Nat. Commun. 2012, 3, 1064. 
[13] A. K. Yadav, C. T. Nelson, S. L. Hsu, Z. Hong, J. D. Clarkson, C. M. Schlepüetz, A. R. Damodaran, P. Shafer, E. Arenholz, L. R. Dedon, D. Chen, A. Vishwanath, A. M. Minor, L. Q. Chen, J. F. Scott, L. W. Martin, R. Ramesh, Nature 2016, 530, 198.

[14] T. Ma, J.-P. Han, IEEE Electron Device Letters 2002, 23, 386.

[15] A. Tagantsev, G. Gerra, J. Appl. Phys. 2006, 100, 051607.

[16] S. Lisenkov, L. Bellaiche, Phys. Rev. B 2007, 76, 020102.

[17] P. Aguado-Puente, J. Junquera, Phys. Rev. B 2012, 85, 184105.

[18] P. Zubko, N. Jeclin, A. Torres-Pardo, P. Aguado-Puente, A. Gloter, C. Lichtensteiger, J. Junquera, O. Stéphan, J.M. Triscone, Nano. Lett. 2012, 12, 2846.

[19] S.M. Nakhmanson, K.M. Rabe, D. Vanderbilt, Phys. Rev. B 2006, 73, 060101; S.M. Nakhmanson, K.M. Rabe, D. Vanderbilt, Appl. Phys. Lett. 2005, 87, 102906.

[20] X. Wu, M. Stengel, K. M. Rabe, D. Vanderbilt, Phys. Rev. Lett. 2008, 101, 087601.

[21] J.H. Lee, J. Yu, U.V. Waghmare, Appl. Phys. Lett. 2009, 105, 016104.

[22] It has been proposed for $\mathrm{BaTiO}_{3}-\mathrm{CaTiO}_{3}$ superlattices (X. Wu, K.M. Rabe, D. Vanderbilt, Phys. Rev. B 2011, 83, 020104) higher ferroelectric polarization in $n=1$ that in $n$ $=2$ due to suppression of $\mathrm{TiO}_{6}$ octahedral distortions in a $\mathrm{CaTiO}_{3}$ layer if either neighboring layer is a $\mathrm{BaO}$ layer, resulting in a ferroelectric instability in $\mathrm{CaTiO}_{3}$.

[23] S.S.A. Seo, H.N. Lee, Appl. Phys. Lett. 2009, 94, 232904.

[24] F. Sánchez, C. Ocal, J. Fontcuberta, Chem. Soc. Rev. 2014, 43. 2272.

[25] A.P. Damodaran, E. Breckenfeld, Z. Chen, S. Lee, L.W. Martin, Adv. Mater. 2014, 26, 6341.

[26] H.N. Lee, S.S. Ambrose Sung, W.S. Choi, C.M. Rouleau, Sci. Reports 2016, 6, 19941.

[27] B. Bein, H.C. Hsing, S.J. Callori, J. Sinsheimer, P.V. Chinta, R.L. Headrick, M. Dawber, Nat. Commun. 2015, 6, 10136. 

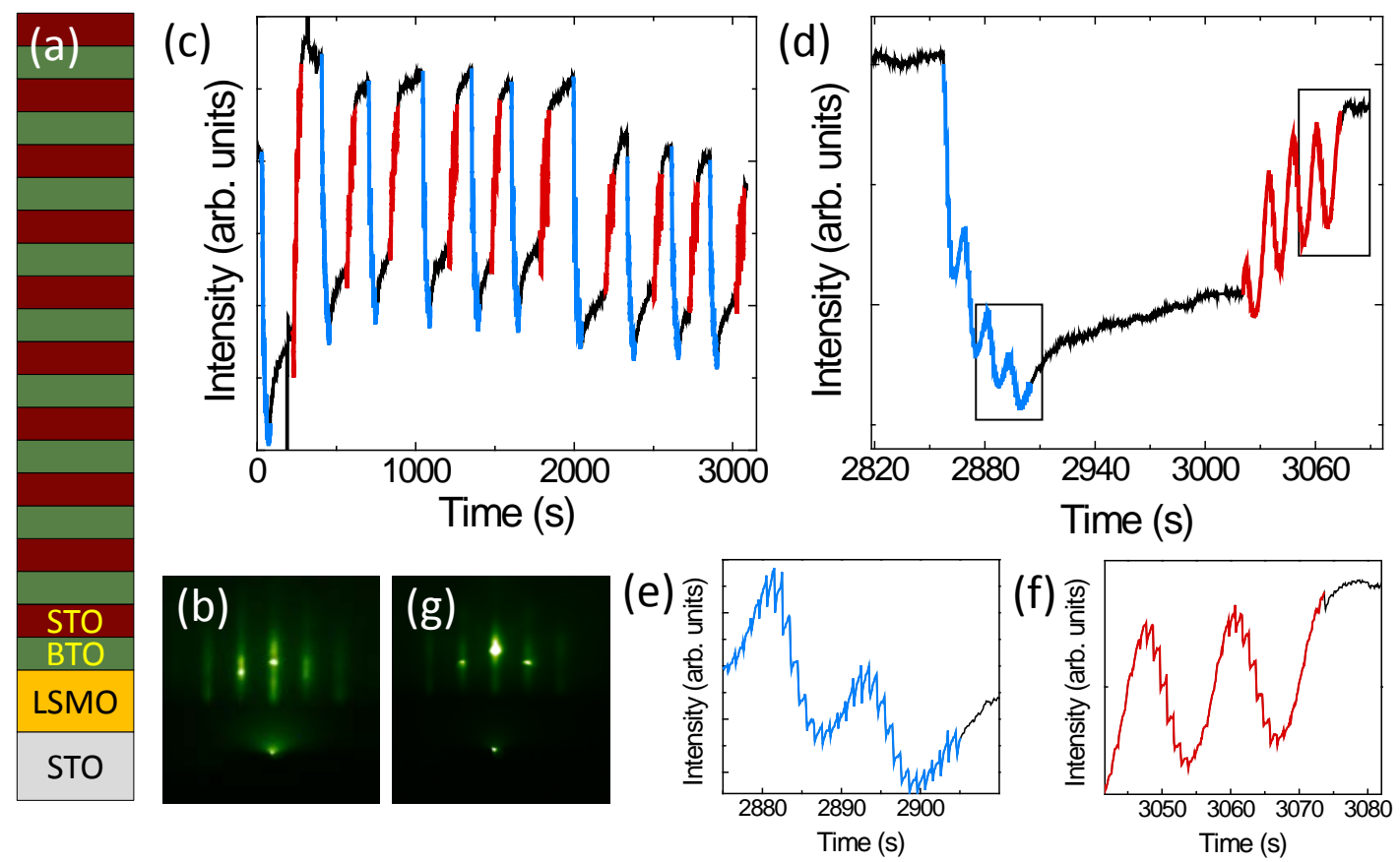

Figure 1. (a) Sketch of a 10x(4-BTO/4-STO) superlattice on LSMO/STO(001). (b) RHEED pattern of the LSMO electrode, recorded along LSMO[100]. (c) Evolution of the RHEED intensity (specular spot) during deposition of the 10x(4-BTO/4-STO) superlattice. The data recorded during BTO and STO growth are in blue and red, respectively. (d) RHEED intensity during the deposition of the $10^{\text {th }}$ superlattice stack. The marked rectangles during BTO and STO growth are zoomed in (e) and (f), respectively. (g) RHEED pattern along STO[110] recorded at the end of the superlattice deposition. 

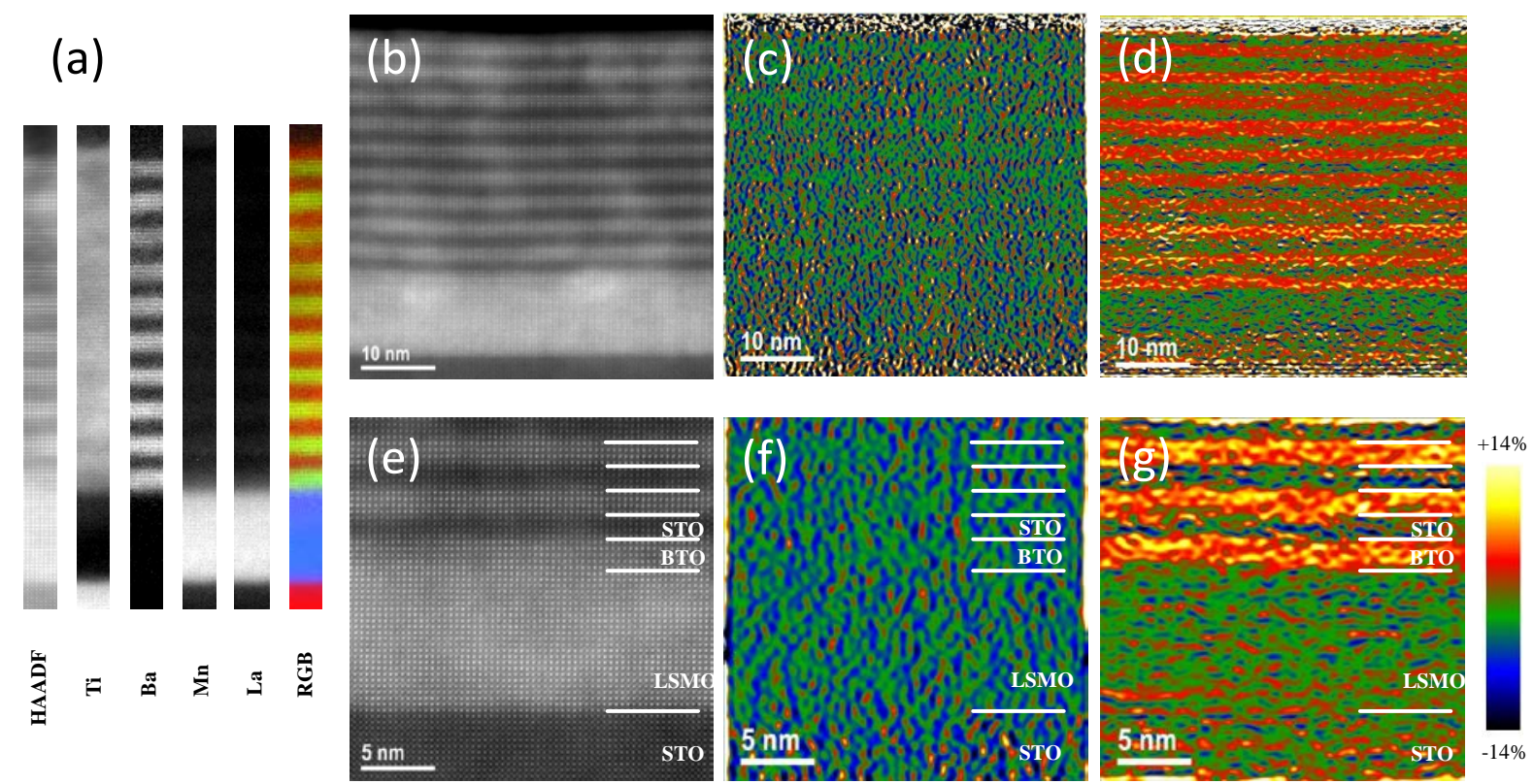

Figure 2. (a) EELS composition maps along a cross-sectional specimen of the 10x(4-BTO/4STO) superlattice, including HAADF and RGB color composition images (Ti in red, Ba in green, $\mathrm{Mn}$ in blue), with the substrate in the bottom of mapped regions. (b) HAADF-STEM image of the cross-section, with the corresponding deformation maps along the in-plane and the out-of-plane directions in (c) and (d), respectively. A zoom of (b) is presented in (e), with the corresponding deformation maps in (f) and (g). 

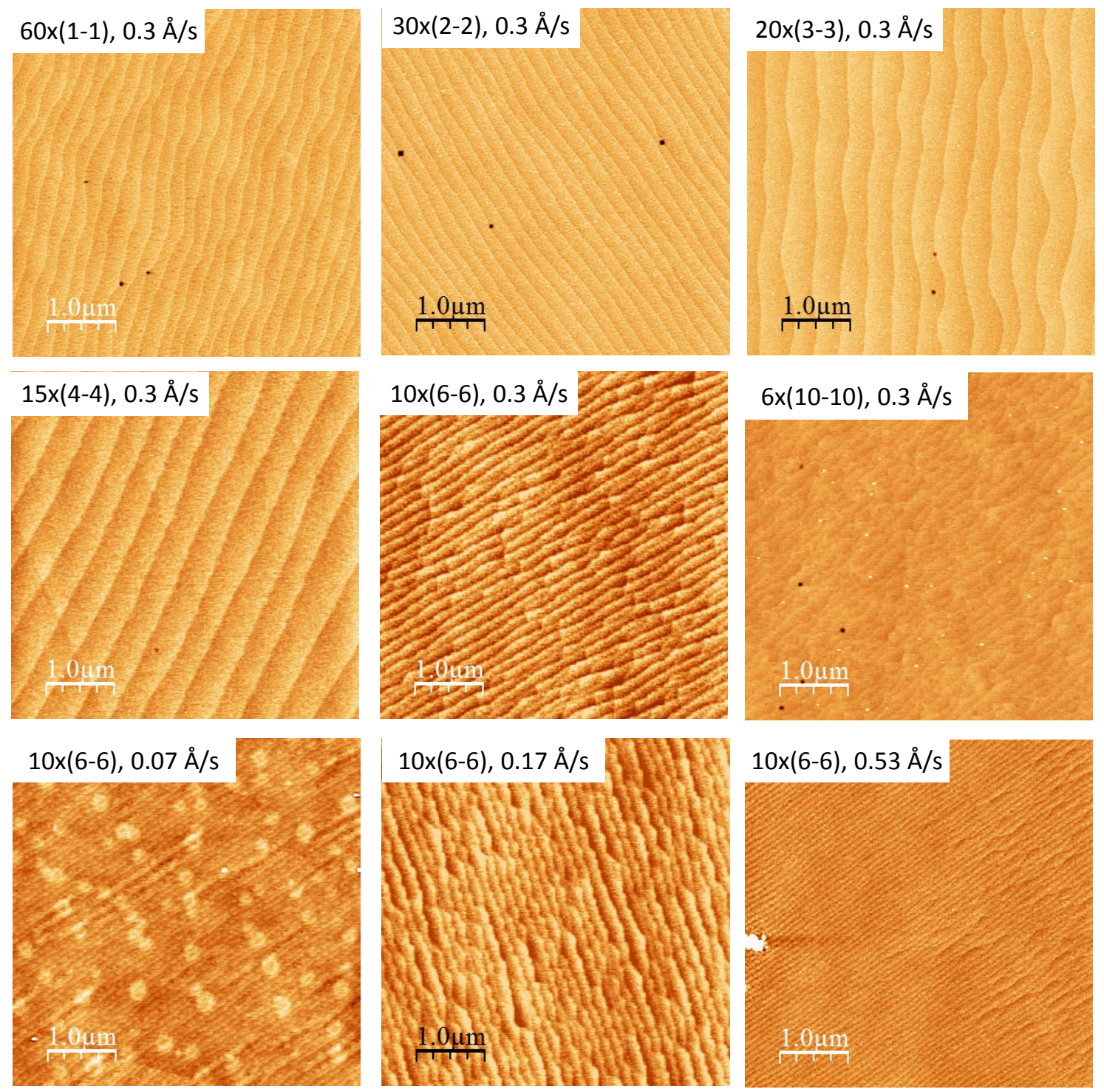

Figure 3. Topographic AFM images $\left(5 \times 5 \mu \mathrm{m}^{2}\right)$ of the $\mathrm{Mx}(\mathrm{n}-\mathrm{BTO} / \mathrm{n}-\mathrm{STO})$ superlattices deposited at a rate of $0.3 \AA$ Apulse and the 10x(6-BTO/6-STO) superlattices deposited at different growth rate. Labels at the top of each image indicate $\mathrm{M}, \mathrm{n}$ and the growth rate. 

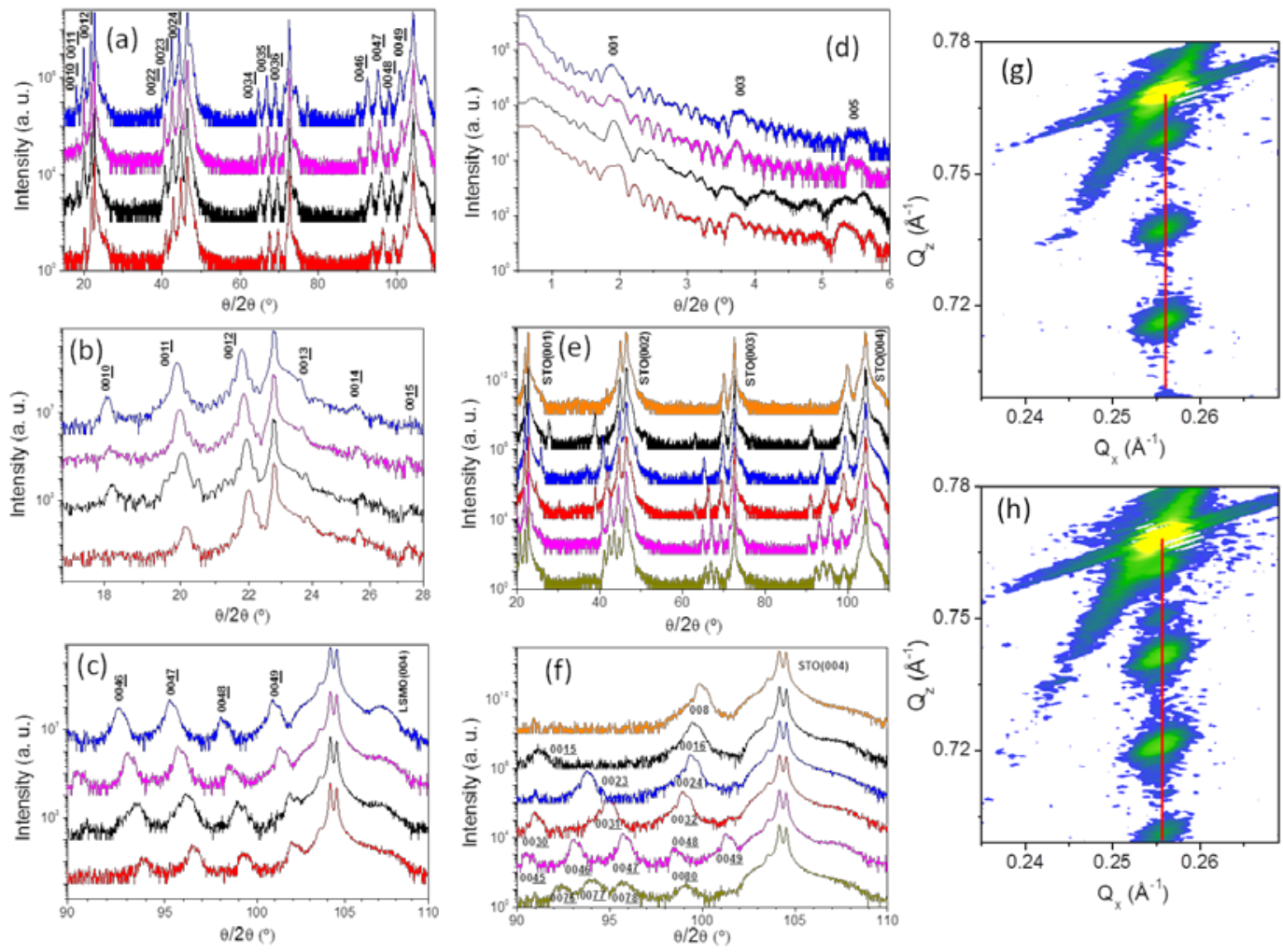

Figure 4. (a) Specular XRD $\theta-2 \theta$ scans of the $10 x(6-B T O / 6-S T O)$ superlattices prepared at different growth rate (Series II). (b) and (c) are zooms of the XRD $\theta-2 \theta$ scans at low and high angles, respectively, and XRR curves are shown in (d). For clarity the curves are shifted vertically, ordered according the growth rate (bottom curve corresponds to the lower growth rate, $0.07 \AA /$ pulse). (e) Specular XRD $\theta-2 \theta$ scans of the $\mathrm{Mx}(\mathrm{n}-\mathrm{BTO} / \mathrm{n}-\mathrm{STO})$ superlattices deposited at a rate of $0.3 \AA$ Ápulse (Series I), with a zoom at high angles in (f). For clarity the curves are shifted vertically, ordered according the number M of BTO-STO bilayers (bottom curve corresponds to the $M=6$ sample, and the top curve to the $M=60$ sample). (g) and (h) show XRD RSM around the STO(103) reflection of the 10x(6-BTO/6-STO) superlattices deposited at growth rate of $0.3 \AA ̊ / p u l s e$ and of $0.07 \AA ̊$ pulse, respectively. 

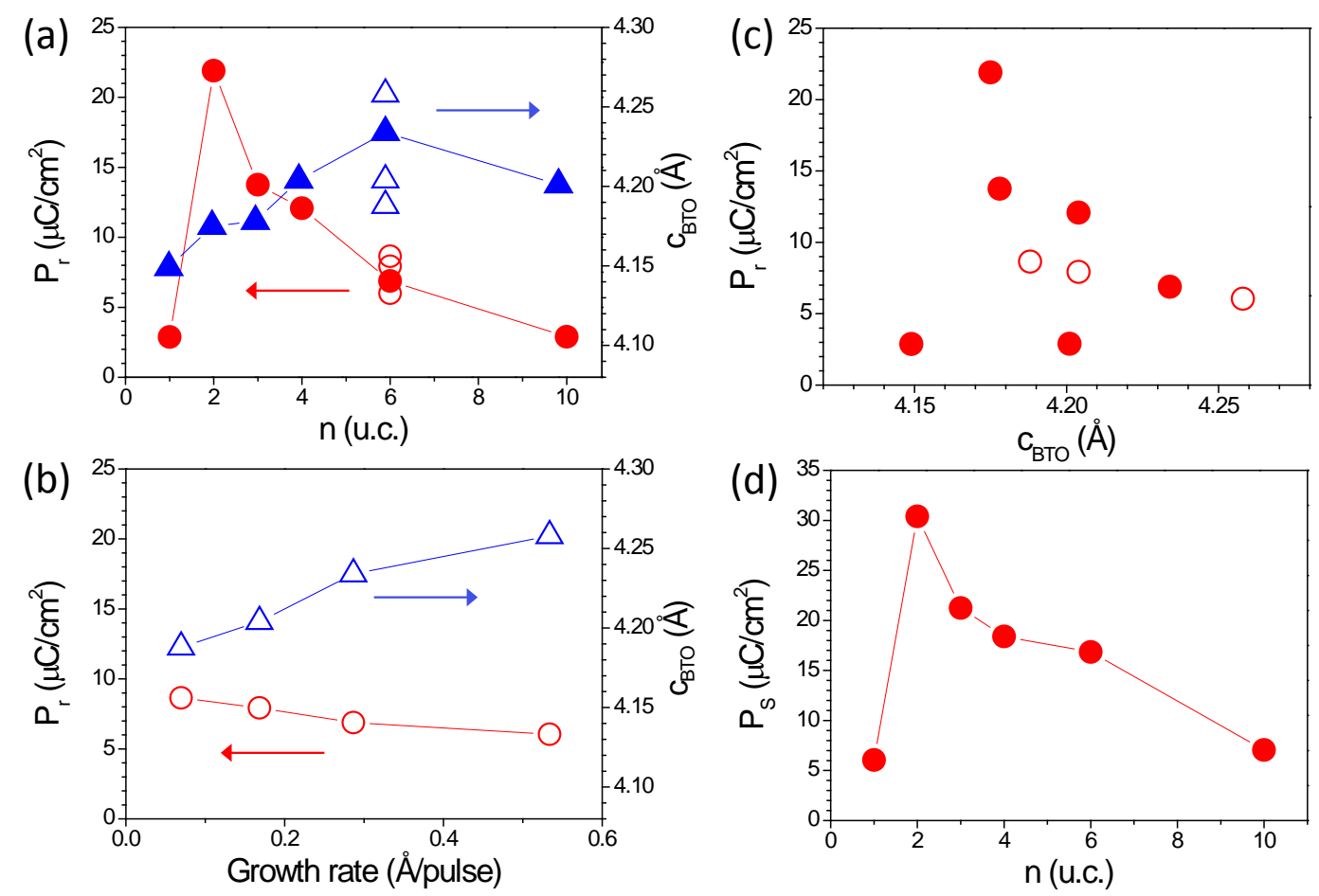

Figure 5. c-axis parameter of BTO (blue triangles) and superlattice remnant polarization (red circles) as a function of (a) $\mathrm{n}$ in $\mathrm{Mx}(\mathrm{n}-\mathrm{BTO} / \mathrm{n}-\mathrm{STO})$ superlattices deposited at $0.3 \AA$ A pulse (Series I), and (b) growth rate in 10x(6-BTO/6-STO) superlattices (Series II). In (a), data corresponding to Series II are included (empty symbols). (c) Superlattice remnant polarization as a function of the c-axis parameter of BTO. (d) Experimental spontaneous polarization of the $\mathrm{Mx}(\mathrm{n}-\mathrm{BTO} / \mathrm{n}-\mathrm{STO})$ superlattices as a function of period $\mathrm{n}$. 

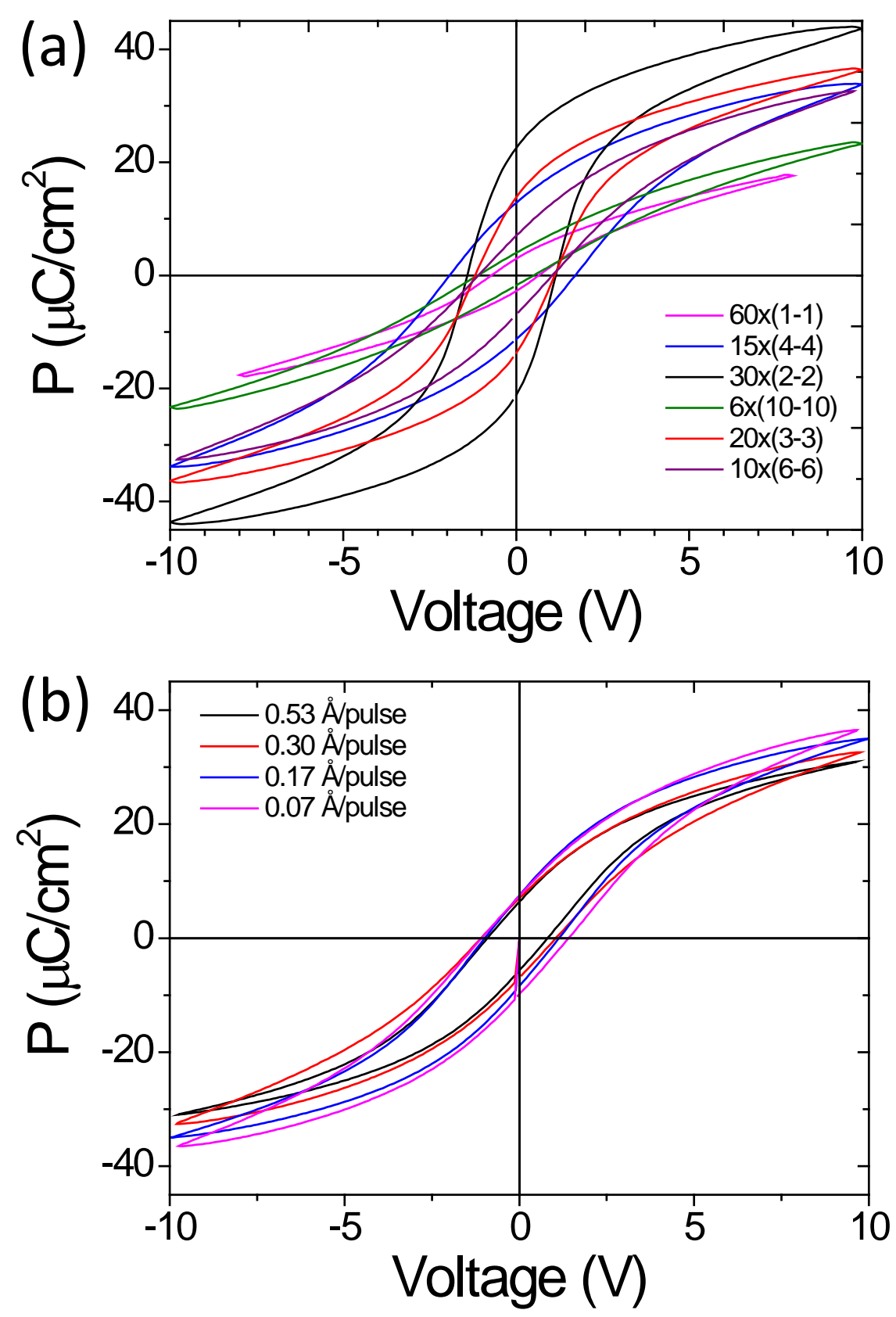

Figure 6. Polarization loops for (a) $\mathrm{Mx}(\mathrm{n}-\mathrm{BTO} / \mathrm{n}-\mathrm{STO})$ superlattices deposited at $0.3 \AA$ A pulse (Series I), and (b) 10x(6-BTO/6-STO) superlattices deposited at different growth rate (Series II). 


\section{$n=2 \quad$ Increasing superlattice period}
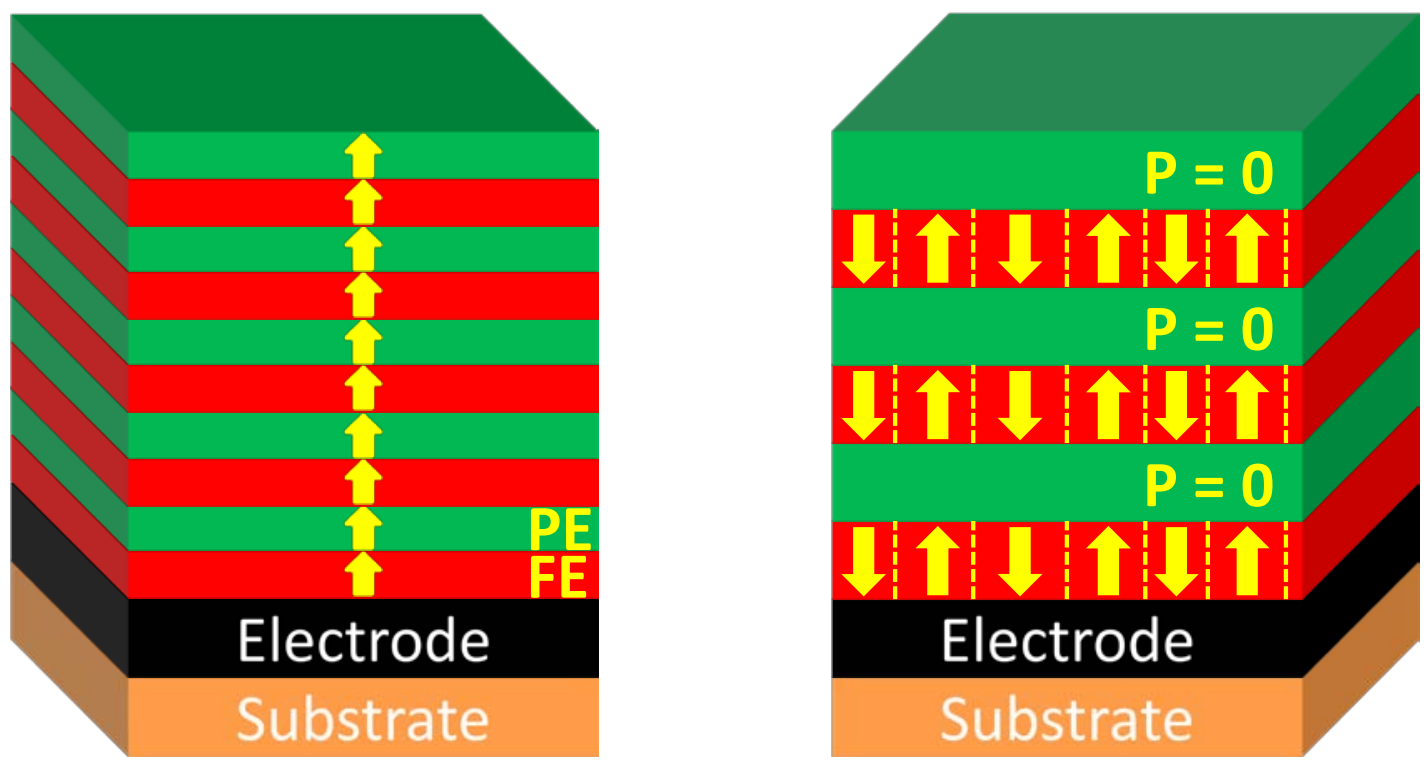

Figure 7. Sketch of possible ferroelectric domains formed in short period (a) and large period (b) Mx(n-BTO/n-STO) superlattices. In short period Mx(n-BTO/n-STO) superlattices uniform polarization across the superlattice can be energetically favored by the low thickness of the STO layers. In contrast polarizing thick STO layers in the large period superlattices can be energetically more costly that the formation of a domain pattern in the ferroelectric BTO layers, causing significant reduction or suppression of switchable ferroelectric response in the superlattice. 
Table 1. Repeated bilayers (M), period (n), number of interfaces and growth rate of the two series of Mx(n-BTO/n-STO) superlattices. The total thickness of all superlattices is fixed to be around $48 \mathrm{~nm}$ (corresponding to 60 u.c. of BTO and 60 u.c. of STO). In Series I (6 samples) the number of BTO-STO interfaces changes from 11 to 119, being the growth rate fixed to $0.30 \AA$ //pulse. In Series II (4 samples) the number of interfaces is fixed to 119 (10x(6BTO/6-STO) superlattices), and the growth rate changes from 0.07 to $0.53 \AA$ Apulse. Note that one of the samples ( $M=10, n=6$, growth rate $=0.30 \AA /$ pulse $)$ is common to both series. All superlattices were deposited on LSMO bottom electrodes in a single process with RHEED monitoring during growth.

\begin{tabular}{|c|c|c|c|c|c|c|}
\hline \multicolumn{7}{|c|}{ Series I } \\
\hline M (units) & 60 & 30 & 20 & 15 & 10 & 6 \\
\hline n (u.c.) & 1 & 2 & 3 & 4 & 6 & 10 \\
\hline Number of interfaces & 119 & 59 & 39 & 29 & 19 & 11 \\
\hline Growth rate (§/pulse) & 0.30 & 0.30 & 0.30 & 0.30 & 0.30 & 0.30 \\
\hline
\end{tabular}

\begin{tabular}{|c|c|c|c|c|}
\hline \multicolumn{5}{|c|}{ Series II } \\
\hline M (units) & 10 & 10 & 10 & 10 \\
\hline n (u.c.) & 6 & 6 & 6 & 6 \\
\hline Number of interfaces & 19 & 19 & 19 & 19 \\
\hline Growth rate $(\AA /$ pulse) & 0.07 & 0.17 & 0.30 & 0.53 \\
\hline
\end{tabular}

